\title{
How to translate neuro-cognitive and behavioural outcome data in animals exposed to paracetamol to the human perinatal setting?
}

\author{
Karel Allegaert ${ }^{1,2,3}$, John van den Anker ${ }^{4,5,6}$
}

\begin{abstract}
${ }^{1}$ Department of Development and Regeneration, KU Leuven, Leuven, Belgium ${ }^{2}$ Department of Pharmaceutical and Pharmacological Sciences, KU Leuven, Leuven, Belgium ${ }^{3}$ Department of Hospital Pharmacy, Erasmus MC, Rotterdam, The Netherlands ${ }^{4}$ Department of Pediatrics, Pharmacology and Physiology, Children's National Medical Center, Washington DC, 20010, USA

5Intensive Care, Erasmus Medical Center-Sophia Children's Hospital, Rotterdam, The Netherlands

${ }^{6}$ Department of Pediatric Pharmacology, University Children's Hospital Basel, Basel, Switzerland
\end{abstract}

Submitted: 5 January 2020

Accepted: 5 May 2020

Arch Med Sci

DOI: https://doi.org/10.5114/aoms.2020.100715

Copyright @ 2020 Termedia \& Banach

\begin{abstract}
Introduction: There are epidemiological - not necessary causal - observations that link perinatal paracetamol (acetaminophen) exposure to impaired neuro-cognition and behaviour, but animal models may assist to better understand the mechanisms.

Material and methods: To provide an overview on preclinical data and mechanisms explored, we conducted a structured literature search on animal models and neuro-cognition and behavioural outcome following perinatal paracetamol exposure.

Results: This search resulted in 20 papers [rat $(n=9)$, zebrafish larvae $(n=6)$, mice $(n=5)$ ], published between 2009 and 2020. Eight discussed pregnancy/ fetal paracetamol exposure, 6 juvenile, 6 studies combined pregnancy and juvenile exposure. Quality assessment (SYRCLE's bias risk) showed a heterogeneous pattern with blinding issues. Most papers $(n=16)$ described paracetamol exposure without indication, except for an induced fever and repetitive needle pricking (rat), brain injury (mice), and a zebrafish nociception model. Reported outcomes related to biochemistry (mono-amines, amino acids, protein expression), anatomy (teratogen, morphology, nuclear size) or behaviour (spatial memory, motor, social behaviour and exploration, sexual behaviour). On mechanisms, the cumulative data support an interesting 'cannabinoid' hypothesis to link paracetamol to neuro-cognitive and behavioural outcome. Besides limited species diversity, there is relevant within-species paracetamol dosing variability (dose, duration) with undocumented exposure. Conclusions: Models should further integrate clinical indications, as nonexposure is the obvious safest setting in the absence of an indication. Besides pain and fever and related to the cannabinoid hypothesis, this should include perinatal brain injury, as there is animal experimental evidence that cannabinoids are neuroprotective in newborn brain injury or asphyxia, further supported by evidence from non-perinatal models of paracetamol-related neuroprotective effects.
\end{abstract}

Key words: paracetamol, juvenile animal model, neurodevelopmental teratology, pregnancy, infant.

\author{
Corresponding author: \\ Karel Allegaert MD, PhD \\ Department of Development \\ and Regeneration \\ Neonatal Intensive Care Unit \\ 49 Herestraat St \\ 3000 Leuven, Belgium \\ Phone: 00-32-16-343210; \\ 00-32-16-230432 \\ Fax: 00-32-16-343211 \\ E-mail: \\ karel.allegaert@uzleuven.be
}




\section{Introduction}

Paracetamol (acetaminophen) has clear analgesic and antipyretic activities, with limited peripheral anti-inflammatory properties when compared to other non-steroidal anti-inflammatory drugs (NSAIDs) such as ibuprofen, diclofenac or indomethacin [1-3]. Both in pregnant women and their newborns, there is sufficient and robust evidence that paracetamol has opioid sparing effects for major pain syndromes, and is effective to treat minor to moderate pain syndromes, or to treat fever with a good short-term safety profile [1].

Paracetamol can be administered by different (enteral or parenteral) routes. As such, it is the drug most commonly used to treat mild to moderate pain or fever, including in pregnant women and during the postpartum period. Recent studies have shown that eight out of ten women take at least one prescription or over-the-counter (OTC) medicine during pregnancy, with paracetamol being the most commonly used OTC drug (65\%) [4]. Oral paracetamol is used to treat mild to moderate pain and fever during pregnancy or labour, while intravenous (IV) paracetamol serves as an effective analgesic in the immediate post-operative period, e.g. after caesarean delivery [5].

Along the same line, effective analgesia is also a crucial and valid part of the care provided to (pre)term neonates. Paracetamol is one of the analgesics to attain this target, with data on drug utilization suggesting that paracetamol is somehow a 'rising star' to handle neonatal pain or fever [6-8]. To further illustrate this, paracetamol prescription has been quantified (14\%) in the EUROPAIN prospective cohort (6680 neonates), and is more frequently prescribed compared to sedatives/hypnotics (12\%) in the neonatal intensive care setting (NICU) [9]. Furthermore, paracetamol also became one of the drugs used to treat (symptomatic) patent ductus arteriosus in preterm neonates [10]. Also, the association between maternal paracetamol use and fetal ductus arteriosus constriction or closure has more recently been reported, although this risk is difficult to quantify and is likely very low $[11,12]$.

Related to this extensive use, paracetamol exposure during pregnancy is perceived to have a good safety/efficacy balance with the lowest risks and greatest benefits [4]. However, there are specific aspects related to pharmacodynamics (PD, intended effects, but also unwanted side effects) that warrant a focused interpretation on the available data, especially during pregnancy (fetal exposure), or when administered in early infancy in (pre)term newborns $[1,6]$. While there are sufficient data on short-term safety, there are still concerns over long-term side-effects following perinatal exposure. These concerns relate to atopy, male fertility, and - most commonly reported - impaired neuro-cognitive and behavioural outcome. These findings are at present mainly based on epidemiological perinatal observations, combined with postulated mechanisms and supported by animal experimental observations [1] However, their main limitation is that epidemiological studies describe associations, not necessarily causal links, and confounding by indication (there is a reason why paracetamol is prescribed) is the most obvious limitation.

Atopy related risks are speculated to be driven by non-selective inhibition by paracetamol on peripheral cyclo-oxygenase (COX) activity. This occurs besides its central nervous system related mechanisms, in a low prostaglandin, low inflammation setting. Due to maturational immunity (lymphocytes, $\mathrm{T}_{\mathrm{H} 1}$ vs. $\mathrm{T}_{\mathrm{H} 2}$ ), this may interfere with antigen tolerance development and may induce atopy $[13,14]$. The mechanism that links perinatal paracetamol exposure to male fertility is claimed to be related to reduced testosterone production $[15,16]$. Fetal paracetamol exposure in the second trimester of pregnancy has been associated with cryptorchidism or hypospadias, while first trimester paracetamol exposure has been associated with a shorter ano-genital distance in male infants $[15,16]$. The currently reported neuro-cognitive and behavioural outcome risks suggested an exposure/effect association and were strongest for hyperactivity and attention deficits [1, 17]. Sug gested mechanisms are related to cerebral inflammation, or to metabolites such as cannabinoids. Masarwa et al. recently reported a systematic review on the link between prenatal paracetamol exposure and the risk for ADHD, autism spectrum disorder (ASD) and hyperactivity, and suggested a pooled risk ratio of 1.34 (95\% Cl: 1.21-1.47), 1.19 (1.14-1.25) and 1.24 (1.04-1.43) respectively, but acknowledged that these observational data remain susceptible to several sources of bias $[18,19]$. In these epidemiological studies, confounding by indication is a relevant issue, as e.g. there is a recent report on the link between maternal fever during pregnancy and attention deficit hyperactivity disorder (ADHD, odds ratio 1.31 and 2.64 for one or at least two fever episodes in the first trimester of pregnancy). Importantly, this risk was neither mitigated nor inflated by paracetamol exposure [20].

The European Medicine Agency (EMA) Pharmacovigilance Risk Assessment Committee (PRAC) recommendation very recently (March 2019) assessed the evidence and concluded that the clinical impact and relevance of these potential associations remains at present still uncertain. Pharmacovigilance Risk Assessment Committee therefore decided not to adapt the existing guid- 
ance, but to add some specific sentences on these associations in the summary of product characteristics (SmPC, the leaflet) in the relevant section on fertility, lactation and pregnancy (adapted wording is written in italics and underlined, copied verbatim from the SmPC). "A large amount of data on pregnant women indicate neither malformative, nor feto/neonatal toxicity. Epidemiological studies on neurodevelopment in children exposed to paracetamol in utero show inconclusive results. If clinically needed, paracetamol can be used during pregnancy however it should be used at the lowest effective dose for the shortest possible time and at the lowest possible frequency" [21].

These uncertainties and the extensive and increasing exposure to paracetamol in perinatal medicine serve as an obvious and urgent call to generate additional data on neuro-cognitive and behavioural outcomes in both human cohorts, as well as juvenile animal models to further explore aspects related to causality, mechanisms or the presence and extent of potential risks. This includes long-term neuro-cognitive and behavioural outcome data in clinical studies, or as part of ongoing studies and cohorts to generate data and create certainties on the existence and extent of any potential negative effects. This can be achieved by introducing a 'pharmacovigilance' concept and approach as part of long-term outcome studies. The paper of Juurjärvi et al. on neuro-cognitive outcome at the age of 2 years in a cohort $(n=48)$ of former preterm $\left(23^{\text {th }}-31^{\text {th }}\right.$ weeks of gestation) neonates initially recruited in a prospective study on prophylactic paracetamol prescription to induce ductus arteriosus closure is a recent example that such data can be generated as secondary outcome reports. In this study, the Griffiths test (IQ score) was similar between exposed and non-exposed cases [22]. Along the same line, albeit within an interventional study and comparing oral paracetamol versus ibuprofen to induce closure of a patent ductus arteriosus in preterm neonates ( $<30$ weeks of gestation), there was no difference in neuro-cognitive outcome between exposed and non-exposed cases at the age of 18-24 months [23].

Besides these pharmacovigilance studies, fetal or juvenile animal studies are another research line or approach to generate additional information on the benefit/risk balance of paracetamol exposure before (fetus) or after delivery (as (pre) term neonate or infant). At best, such studies should also include the indication to administer paracetamol to consider simultaneously both exposure and indication. In a chronic inflammatory pain and morphine juvenile rat model, it was concluded that persistent pain (formalin model) and morphine treatment in a neonatal rat affected the long-term behavioural outcome in adulthood.
More relevantly, some of these outcomes were attenuated when both (opioid administration to treat the formalin-induced pain) were co-administered [24].

Based on our background in perinatal clinical pharmacology in humans, we conducted a structured search on the currently reported fetal or juvenile animal models following paracetamol exposure to provide a qualitative and quantitative overview on preclinical data.

\section{Material and methods}

A structured search was performed on December 26, 2019, using a broad search strategy [(paracetamol) AND (rat or rats or mouse or mice or murine or animal or monkey or macaque or nonhuman)) AND (neurodevelopmental or adhd or mental or social cognition or brain or behaviour or memory or neurotoxic or autis)] in PubMed to retrieve the available preclinical studies related to paracetamol exposure and the topic of interest (neuro-cognitive and behavioural outcome, perinatal exposure). Subsequent inclusion criteria for the final dataset of studies were any topics related to fetal or juvenile animal studies on neurotoxicity, neuro-cognition or behavioural outcome or any other indicator of relevance to the nervous system, based on title, abstract and full paper. The list of titles was screened twice to ensure full and accurate retrieval, and uncertainties were discussed (KA, JvdA). Once the list was established, a reference check and citation verification (on PubMed and on the journal website) were performed for the included studies.

In an attempt to assess the quality of the pre-clinical studies, the risk of bias was assessed with SYRCLE's risk of bias tool for preclinical studies [25]. This was performed by KA, and uncertainties were discussed with JvdA until consensus was reached. Because we anticipated a heterogeneous pattern in species, interventions and outcome reported, a data extraction form was used to collect the data on study population (number, species, age, gender), intervention (paracetamol exposure, duration, indication), and outcome to provide a quantitative, descriptive overview.

\section{Results}

The above-mentioned search strategy resulted in 2610 hits in PubMed for screening. Based on title screening, 128 potential relevant hits were retained, to result in 21 potential hits after abstract assessment. Two additional papers were excluded after reading the full paper version (in both because paracetamol was not used in these studies, but mentioned in the abstract), and one additional paper was included following citation 


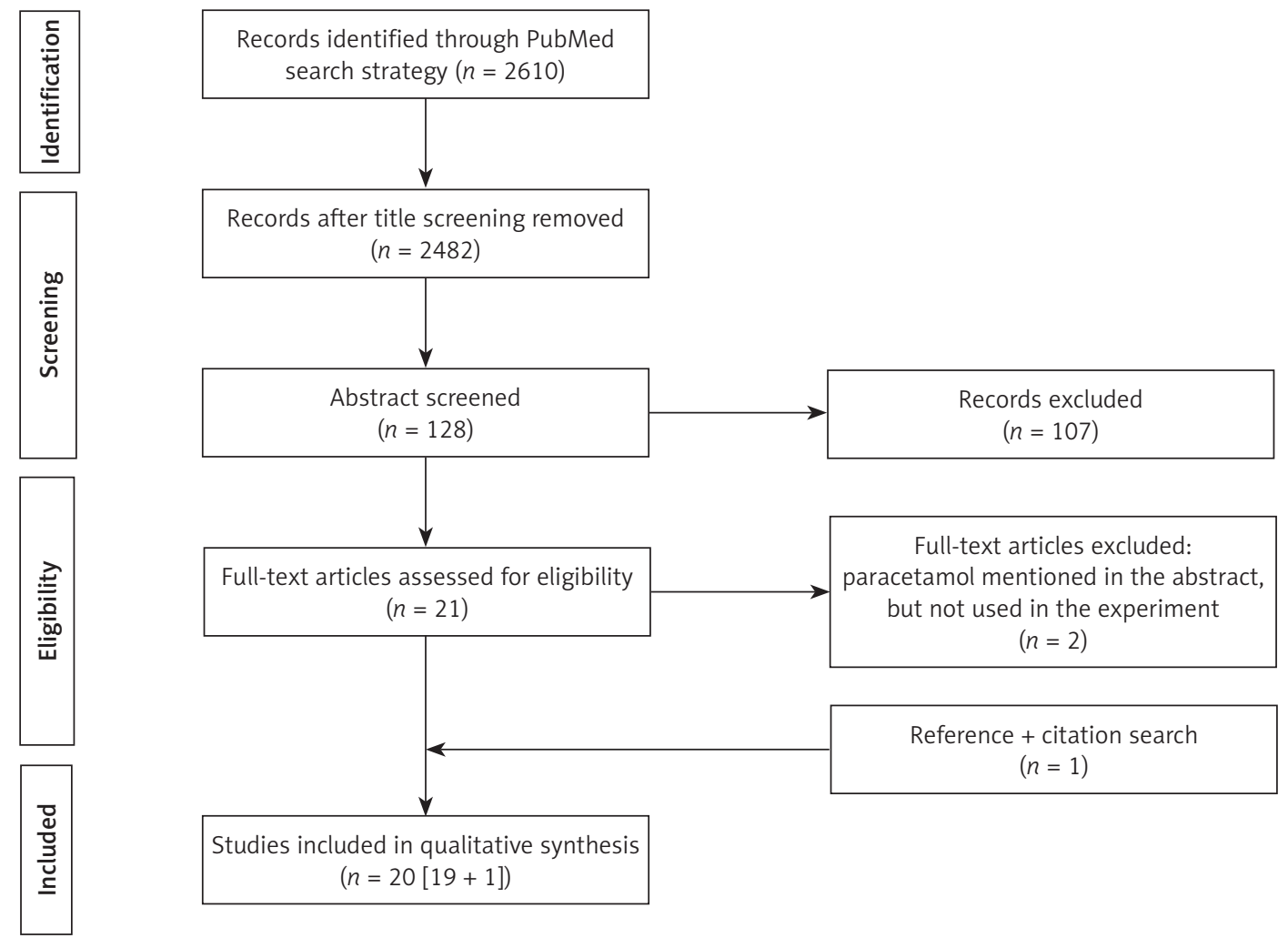

Figure 1. PRISMA flow chart describing the search strategy and results

verification to result in 20 papers (Prisma flowchart, Figure 1).

Table I provides an overview on the quality assessment (SYRCLE's risk of bias tool) results [26-45]. This reflects a heterogeneous pattern with mainly issues related to blinding (for performance, for detection) procedures in a relevant portion of the studies (unclear or unreported, high risk in 16/20 for both) and allocation concealment (was the method used to conceal the allocation sequence described in sufficient detail to determine whether intervention allocation could have been foreseen before or during enrolment?). Power calculations were not reported in any of the studies. This does not mean that these aspects were not considered during the design or study conduct, but we were not able to extract this information from the methods section description. Table II provides an overview on the characteristics of these studies [26-45]. Juvenile species reported are rat $(n=9)$, zebrafish larvae $(n=6)$, or mice/mouse $(n=5)$. Eight studies discussed embryological or fetal aspects of paracetamol exposure, 6 reported on juvenile animals (postnatal, but equivalent to (pre)term human age), while 6 studies combined pregnancy and juvenile exposure in the same animal. Within a given animal model, there is relevant variability in paracetamol dosing, while exposure has not been quantified. Paracetamol exposure in the zebrafish or rat models varied between the different research groups and studies.

The majority $(n=16)$ of the studies retained in the analysis were based on paracetamol exposure without indication for exposure, except for the induced fever model and the repetitive needle pricking model (both in rats), the excitotoxic brain injury model (mouse), and the zebrafish larvae nociception model.

Outcomes relate to biochemical alterations (mono-amines, amino acids, protein expression in the central nervous system), anatomical (teratogen, morphology, nuclear size) or behavioural (spatial memory, motor performance, social behaviour and exploration, sexual behaviour) aspects. Both within as well as between different animal models, the outcome was not always 'uniform'. To illustrate this, BDNF is 2-fold lower (rat = striatum), similar (rat = prefrontal cortex, hippocampus; mice $=$ hippocampus) or higher $($ mice $=$ frontal and parietal).

\section{Discussion}

With this structured search, we aimed to provide an overview on the currently available animal models on neuro-cognitive and behavioural aspects following paracetamol exposure, with the a priori intention to reflect on their applicability for the human perinatal setting. This is because these animal experimental studies can be of relevance to explore aspects related to causal mecha- 


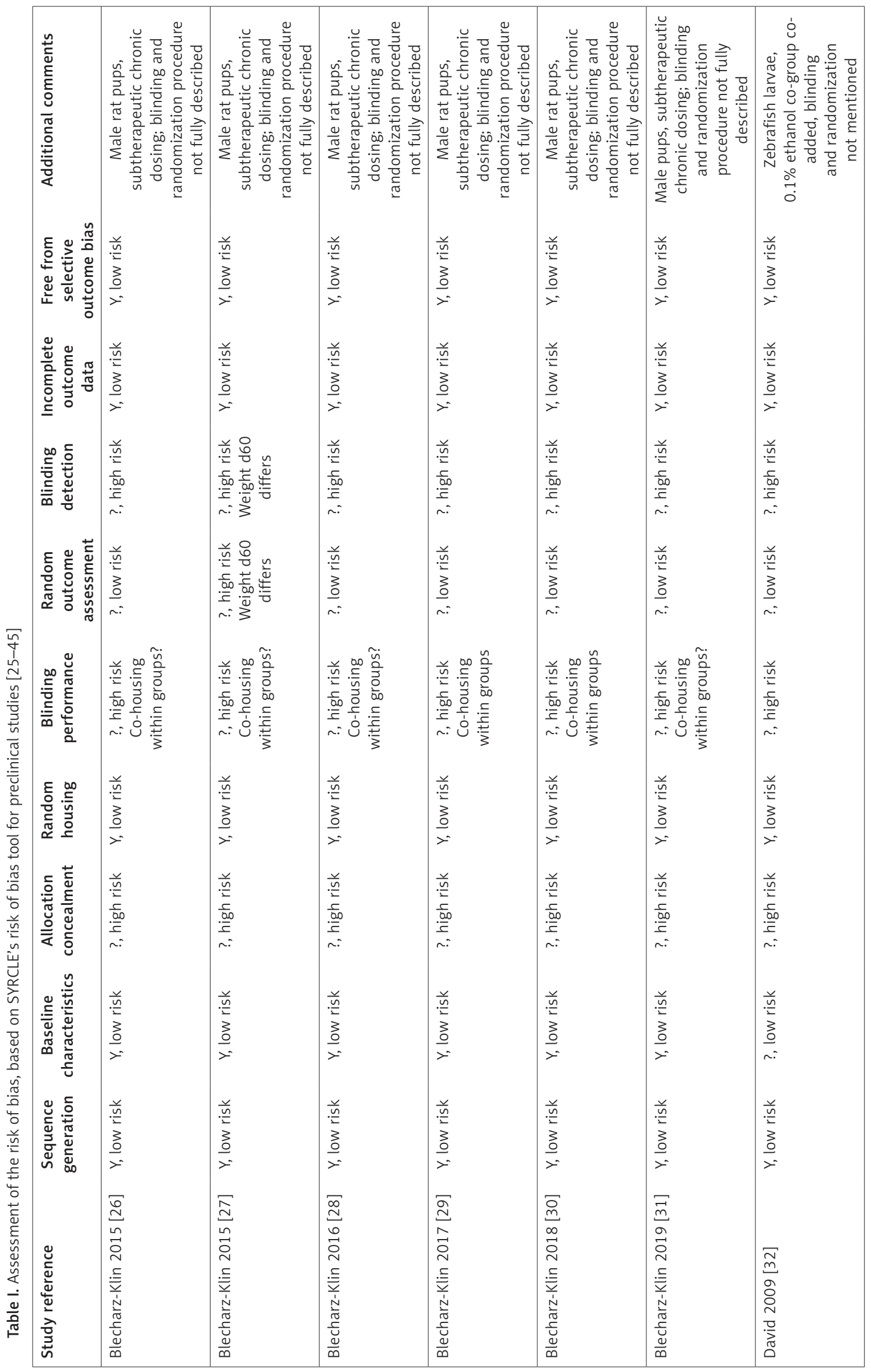




\begin{tabular}{|c|c|c|c|c|c|c|c|}
\hline 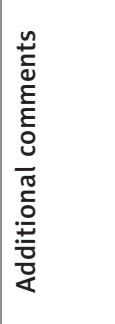 & 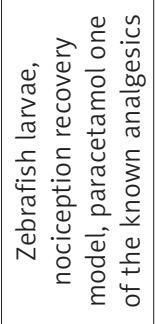 & 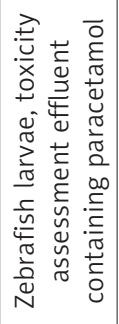 & 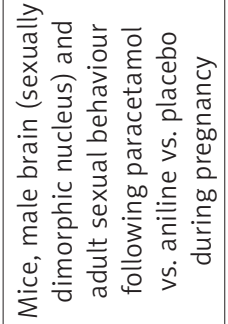 & 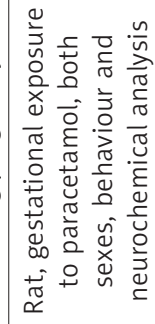 & 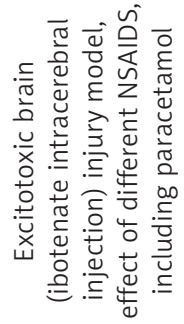 & 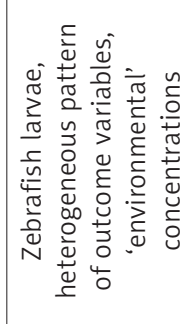 & 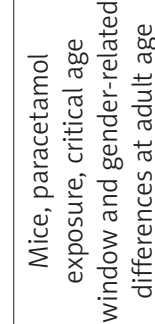 \\
\hline 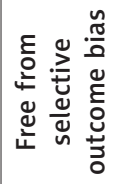 & 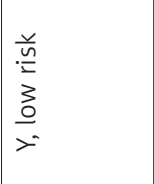 & $\begin{array}{l}\frac{.}{\frac{.0}{2}} \\
\frac{3}{3} \\
\frac{0}{\nu}\end{array}$ & 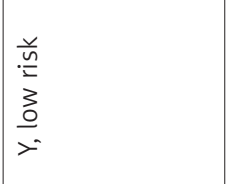 & 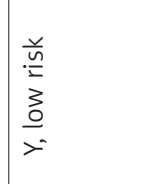 & $\begin{array}{l}\frac{0}{3} \\
\frac{0}{2} \\
\frac{0}{2} \\
>\end{array}$ & 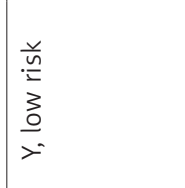 & 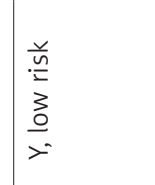 \\
\hline 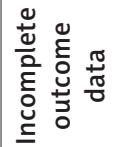 & 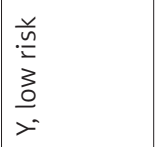 & 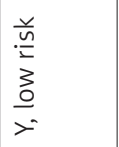 & 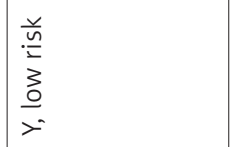 & $\begin{array}{l}\frac{y}{\underline{m}} \\
\frac{.}{3} \\
\frac{0}{x}\end{array}$ & 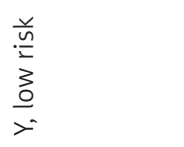 & 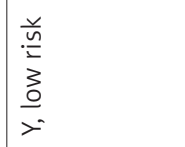 & 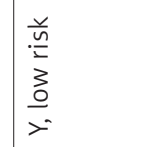 \\
\hline 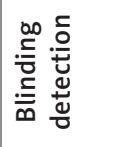 & 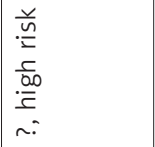 & 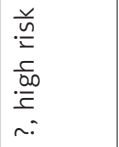 & 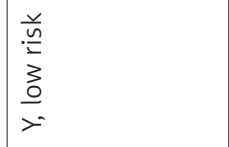 & 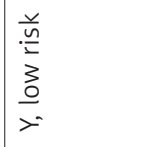 & 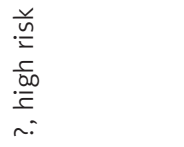 & 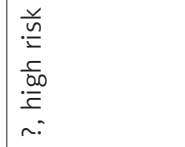 & 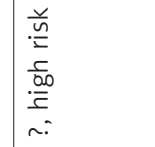 \\
\hline 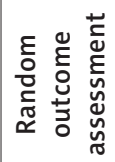 & $\mid \begin{array}{l}\frac{.}{2} \\
\frac{0}{2} \\
3 \\
0 \\
n:\end{array}$ & 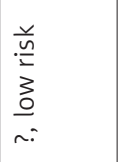 & 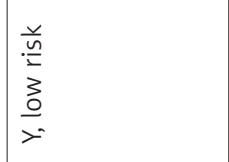 & $\begin{array}{l}\frac{y}{2} \\
\frac{3}{2} \\
\frac{3}{x} \\
\end{array}$ & 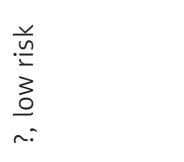 & 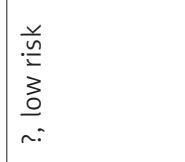 & 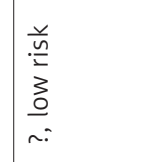 \\
\hline 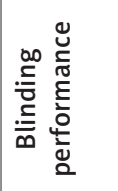 & 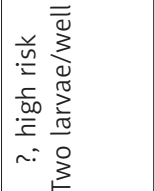 & 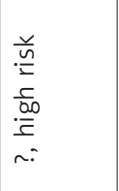 & 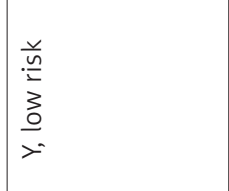 & $\begin{array}{l}\frac{y}{2} \\
\frac{3}{3} \\
\frac{3}{x} \\
\end{array}$ & 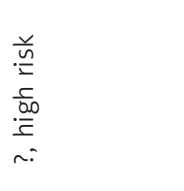 & 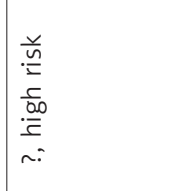 & 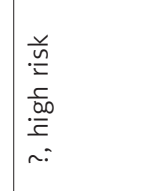 \\
\hline 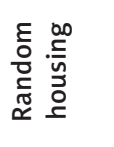 & 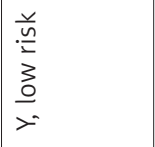 & 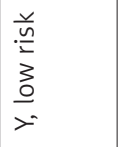 & 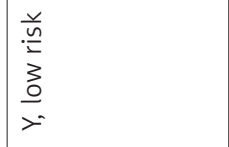 & 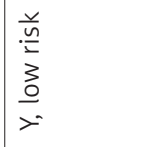 & $\begin{array}{l}\frac{0}{\frac{0}{2}} \\
\frac{3}{2} \\
\frac{2}{2}\end{array}$ & $\begin{array}{l}\frac{y}{-3} \\
\frac{3}{2} \\
\underline{3} \\
>=\end{array}$ & 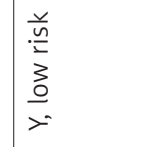 \\
\hline 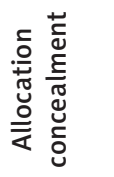 & 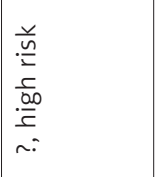 & 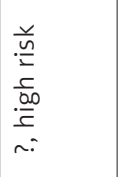 & 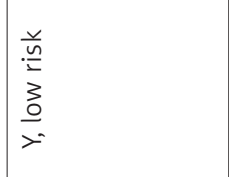 & 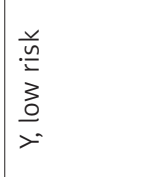 & 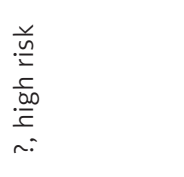 & 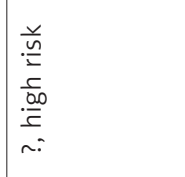 & 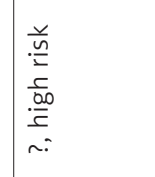 \\
\hline 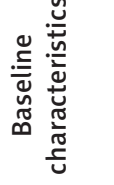 & 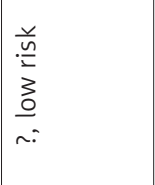 & $\begin{array}{l}\frac{\breve{n}}{2} \\
\frac{3}{x} \\
\frac{3}{x}\end{array}$ & 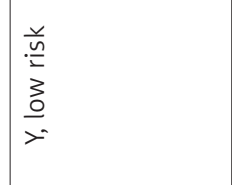 & $\begin{array}{l}\frac{y}{2} \\
\frac{3}{2} \\
\frac{3}{\nu}\end{array}$ & $\begin{array}{l}\frac{.}{2} \\
\frac{3}{2} \\
\frac{3}{x}\end{array}$ & 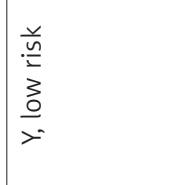 & 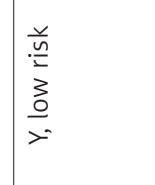 \\
\hline 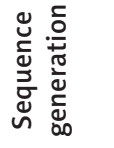 & 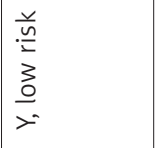 & 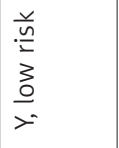 & 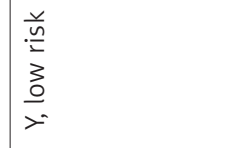 & 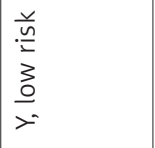 & $\begin{array}{l}\frac{.0}{2} \\
\frac{0}{2} \\
\frac{0}{x}\end{array}$ & 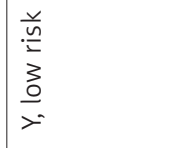 & 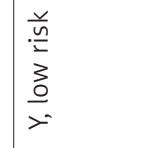 \\
\hline 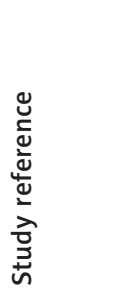 & 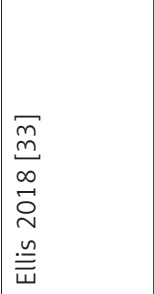 & 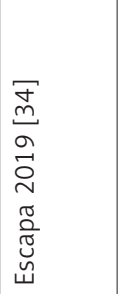 & 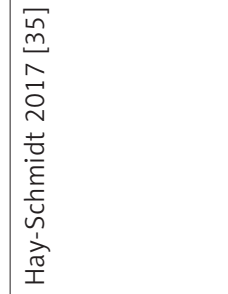 & 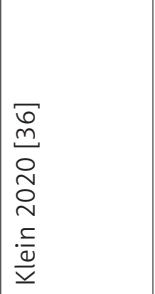 & 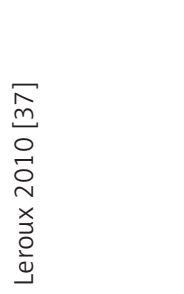 & 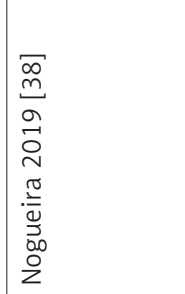 & 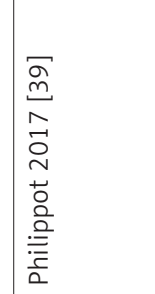 \\
\hline
\end{tabular}




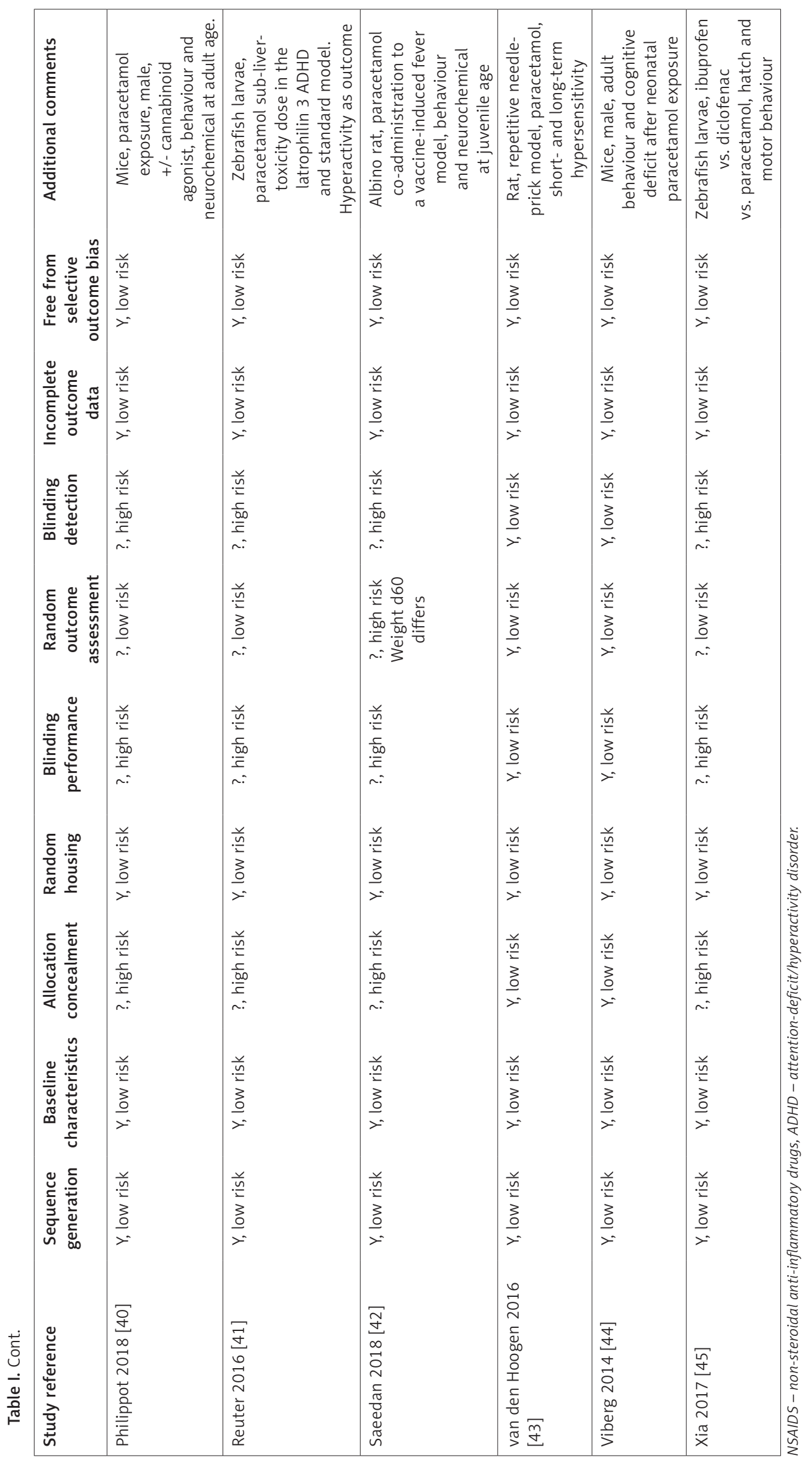




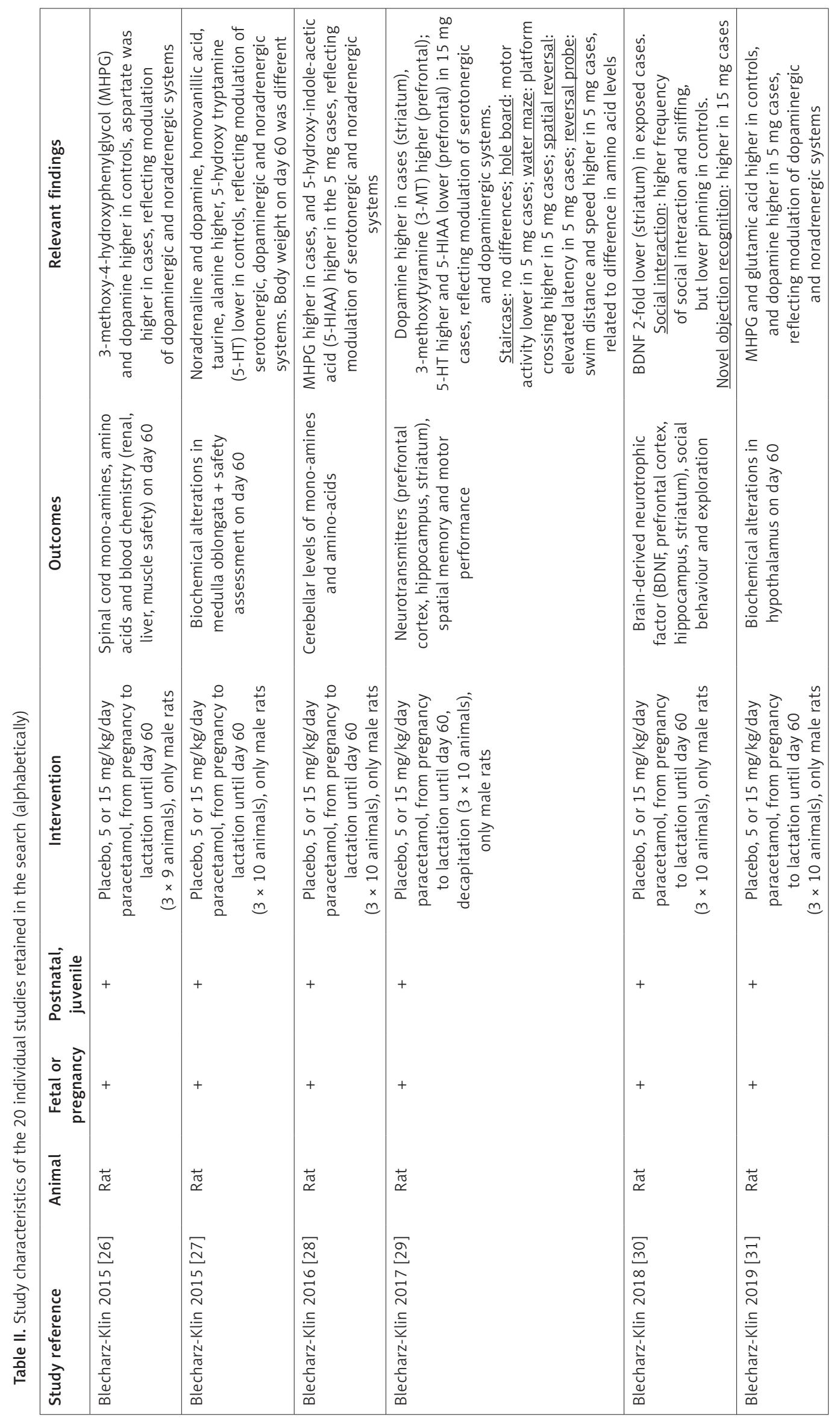




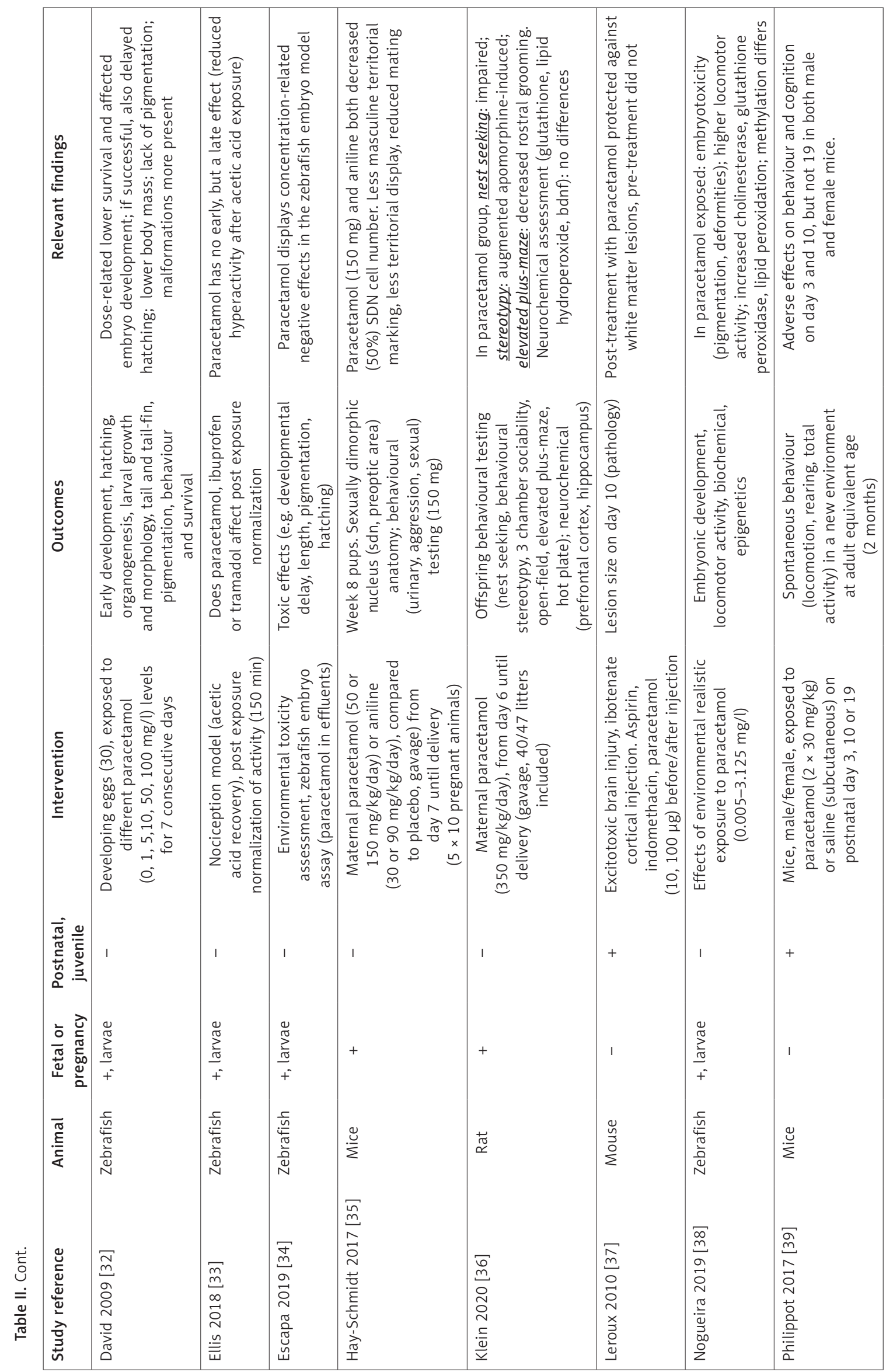




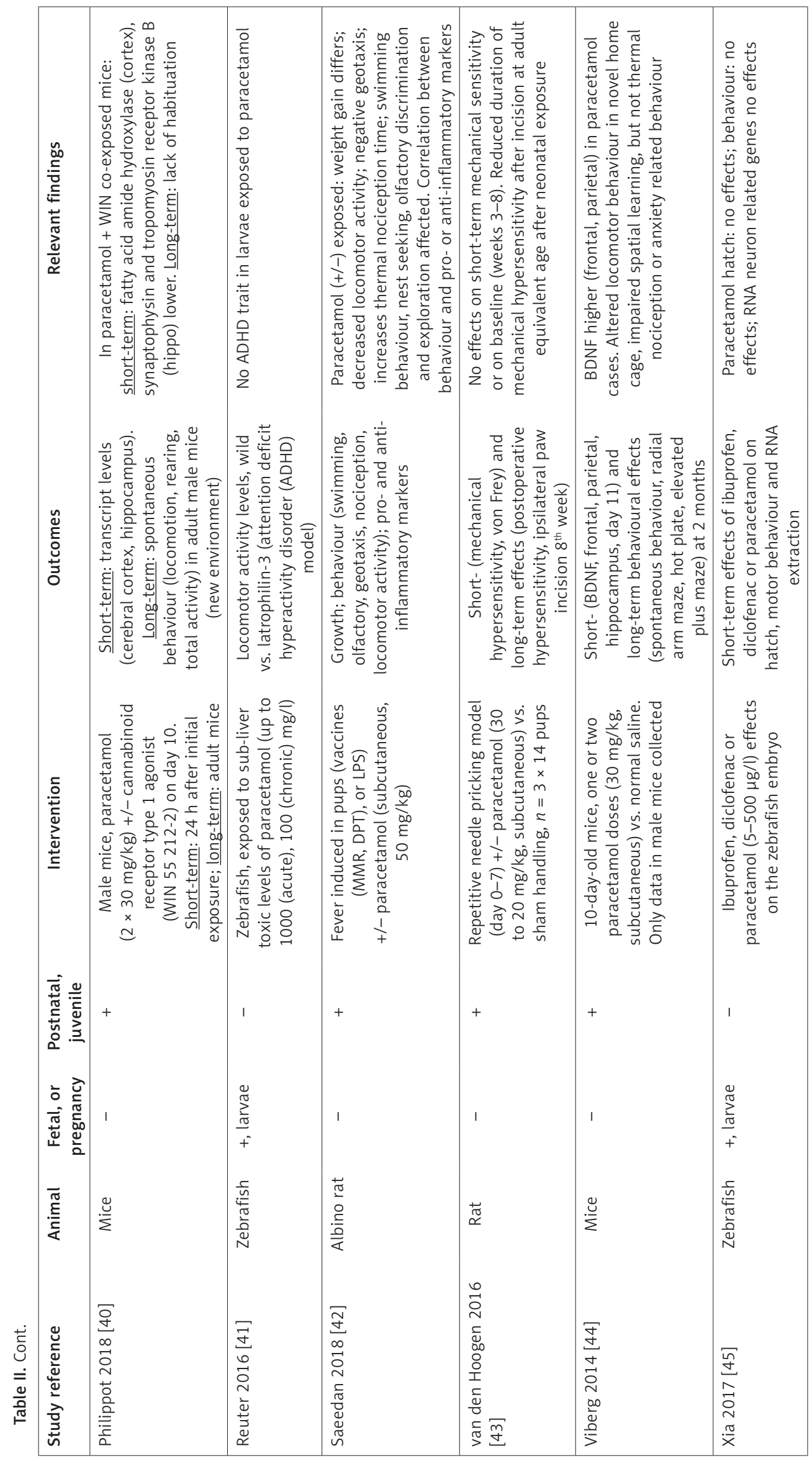


nisms or the presence and extent of potential risks for the perinatal human setting. In this way, these models may assist to close the gap between epidemiological association and causality.

Related to the causal mechanism, the cumulative data generated in juvenile animals provide support for an interesting 'cannabinoid' hypothesis to link paracetamol exposure and neuro-cognitive and behavioural impaired outcome. Pregnancy (maternal, fetal) and preterm specific aspects of paracetamol metabolism have been described $[5,46]$. Paracetamol drug metabolism also includes an active metabolite ( $p$-aminophenol) that interacts with cannabinoid receptors. Related to this, paracetamol and $\Delta(9)$-tetrahydrocannabinol, but not ibuprofen, resulted in developmental neurotoxicity in a mouse model $[39,40,47]$. A single injection of $\Delta(9)$-tetrahydrocannabinol on postnatal day 10 altered adult spontaneous behaviour and habituation rates in adult mice, similar to the pattern observed following acetaminophen exposure [40, 47, 48]. Furthermore, co-administration of a cannabinoid receptor agonist enhanced the developmental neurotoxicity of paracetamol in the same mouse model [40]. This suggests that the experimental animal and perinatal epidemiological research lines in humans on cannabinoids and paracetamol should further interact and liaise [49]. An alternative hypothesis relates to reduction of cerebral COX-activity as there is evidence that COX-2 polymorphisms (affecting phenotypic COX activity) explain in part the differences in cognitive outcome in former preterm neonates [50]. However, ibuprofen in the same mouse model did not result in the developmental neurotoxicity [47].

The overview provides a heterogeneous pattern of studies on neuro-cognitive and behavioural outcome following paracetamol administration in a limited number of small species, but without data in 'higher animals' such as the rabbit, or when very focussed, topical questions are raised, sheep or monkey. Neuropathological and -behavioural consequences of preterm birth have recently been described in the rabbit, and this model has very recently been applied to quantify the effects of caffeine exposure [51, 52]. For sheep, a chronically catheterized fetal sheep model to assess effects of non-narcotic analgesics has been described, while this species has been used to assess the impact of steroids on the developing brain $[53,54]$. As a final illustration, the fetal neurotoxicity of dexmedetomidine has been studied in a pregnant cynomolgus monkey model, as well as a paracetamol hepatotoxicity model in the same species $[55,56]$.

Besides diversification in species, there are 3 other aspects that we feel - driven by our background in perinatal clinical pharmacology in humans - may be of relevance to further consider the benefit/risk balance. First, interspecies similarities in paracetamol dose/exposure should be further assessed. The variability in paracetamol dosing for the different animals in the absence of data on exposure makes it difficult to retrieve the signal or trend and link pharmacokinetics to pharmacodynamics (Table II). Second, we should further discriminate between acute $(<1$ week human equivalent age) and chronic exposure. Related to this, animal models that collect data following chronic and ongoing exposure (like the Blecharz-Klin model) during sample collection have their scientific value. However, such a design makes it difficult to disentangle the effects related to the acute (ongoing) and chronic exposure.

Finally, the current models do not yet reflect the clinical setting as indications should be integrated in these models. It is quite obvious that non-exposure is the safest setting if there is no indication to administer paracetamol, but safety/efficacy assessment is a balanced exercise considering both indication (pain, fever, ductus arteriosus) and exposure. To further illustrate this, we refer to the Leroux, Saeedan and van den Hoogen models that assessed the impact of paracetamol integrated in a perinatal excitotoxic brain injury, a fever and a procedural pain model, respectively. Such models are closer to the earlier mentioned example of the effects of opioids in an inflammatory pain model [24]. There are some examples of such animal experimental design in the non-perinatal age window to assess neurological effects of paracetamol. Paracetamol reduces mitochondrial dysfunction during early cerebral post-ischemic reperfusion in rats [57]. Within the same topic, paracetamol potentiates endothelin-A receptor antagonist BQ123 induced hypothermia and reduces infarction following focal cerebral ischemia in rats [58]. Interestingly, and related to the cannabinoid hypothesis mentioned as a mechanism of toxicity, there is animal experimental evidence that cannabinoids are neuroprotective in perinatal asphyxia and newborn brain injury [59]. This suggests that it is worth the effort to explore the pharmacokinetics and -dynamics of paracetamol in perinatal asphyxia animal models and when successful - in newborns undergoing whole body hypothermia. At least, this suggests that the toxicity of paracetamol should be explored beyond the well-known hepatotoxicity in children [60]. Along the same line, neuro-behavioural outcome should be included as a long-term outcome parameter of interest when paracetamol is prescribed to preterm neonates for e.g. patent ductus arteriosus during respiratory adaptation [10, 61].

Based on the structured search on perinatal animal models and neuro-cognitive and behavioural outcome following paracetamol exposure, we conclude that there is still limited species diversity with 
absence of 'higher' animal models. Furthermore, there is relevant within-species paracetamol dosing variability (dose, duration) with undocumented exposure. Based on our background in perinatal human clinical pharmacology, we highly recommend that these models should further integrate clinical indications, as non-exposure is the obvious safest setting in the absence of an indication. Besides pain and fever and related to the cannabinoid hypothesis, this should include perinatal brain injury, as there is animal experimental evidence that cannabinoids are neuroprotective in newborn brain injury or asphyxia, further supported by evidence in non-perinatal models of paracetamol-related neuroprotective effects.

\section{Conflict of interest}

The authors declare no conflict of interest.

\section{References}

1. Allegaert K, van den Anker JN. Perinatal and neonatal use of paracetamol for pain relief. Semin Fetal Neonatal Med 2017; 22: 308-13.

2. Pacifici GM, Allegaert K. Clinical pharmacology of paracetamol in neonates: a review. Curr Ther Res Clin Exp 2014; 77: 24-30.

3. Anderson BJ. Paracetamol (acetaminophen): mechanisms of action. Paediatr Anaesth 2008; 18: 915-21.

4. Ceulemans M, Lupattelli A, Nordeng H, Odalovic M, Twigg M, Foulon V. Women's beliefs about medicines and adherence to pharmacotherapy in pregnancy: opportunities for community pharmacists. Curr Pharm Des 2019; 25: 469-82.

5. Mian P, van den Anker JN, van Calsteren K, et al. Physiologically based pharmacokinetic modeling to characterize acetaminophen pharmacokinetics and N-Acetyl-p-Benzoquinone imine (NAPQI) formation in non-pregnant and pregnant women. Clin Pharmacokinet 2020; 59: 97-110.

6. Cuzzolin L, Antonucci R, Fanos V. Paracetamol (acetaminophen) efficacy and safety in the newborn. Curr Drug Metab 2013; 14: 178-85.

7. Allegaert K, Simons S, van den Anker J. Research on medication use in the neonatal intensive care unit. Expert Rev Clin Pharmacol 2019; 12: 343-53.

8. Hopchet L, Kulo A, Rayyan M, et al. Does intravenous paracetamol administration affect body temperature in neonates? Arch Dis Child 2011; 96: 301-4.

9. Carbajal R, Eriksson M, Courtois E, et al. Sedation and analgesia practices in neonatal intensive care units (EUROPAIN): results from a prospective cohort study. Lancet Respir Med 2015; 3: 796-812.

10. Allegaert K, Anderson B, Simons S, van Overmeire B. Paracetamol to induce ductus arteriosus closure: is it valid? Arch Dis Child 2013; 98: 462-6.

11. Dathe K, Frank J, Padberg S, et al. Negligible risk of prenatal ductus arteriosus closure or fetal renal impairment after third-trimester paracetamol use: evaluation of the German Embryotox cohort. BJOG 2019; 126: 1560-7.

12. Allegaert K, Mian P, Lapillonne A, van den Anker JN. Maternal paracetamol intake and fetal ductus arteriosus constriction or closure: a case series analysis. $\mathrm{Br} J \mathrm{Clin}$ Pharmacol 2019; 85: 245-51.
13. Langhendries JP, Allegaert K, van den Anker JN, Veyckemans F, Smets F. Possible effects of repeated exposure to ibuprofen and acetaminophen on the intestinal immune response in young infants. Med Hypotheses 2016; 87: 90-6.

14. Eyers S, Weatherall M, Jefferies S, Beasley R. Paracetamol in pregnancy and the risk of wheezing in offspring: a systematic review and meta-analysis. Clin Exp Allergy 2011; 41: 482-9.

15. Fisher BG, Thankamony A, Hughes IA, Ong KK, Dunger DB, Acerini CL. Prenatal paracetamol exposure is associated with shorter anogenital distance in male infants. Hum Reprod 2016; 31: 2642-50.

16. Kilcoyne KR, Mitchell RT. Assessing the impact of inutero exposures: potential effects of paracetamol on male reproductive development. Arch Dis Child 2017; 102: 1169-75.

17. Bauer AZ, Kriebel D, Herbert MR, Bornehag CG, Swan SH. Prenatal paracetamol exposure and child neurodevelopment: a review. Horm Behav 2018; 101: 125-47.

18. Masarwa R, Levine H, Gorelik E, Reif S, Perlman A, Matok I. Prenatal exposure to acetaminophen and risk for attention deficit hyperactivity disorder and autistic spectrum disorder: a systematic review, meta-analysis, and meta-regression analysis of cohort studies. Am J Epidemiol 2018; 187: 1817-27.

19. Damkier P, Scialli AR, Lusskin SI. Acetaminophen in pregnancy and adverse childhood neurodevelopment. JAMA Pediatr 2017; 171: 396.

20. Gustavson K, Ask H, Ystrom E, et al. Maternal fever during pregnancy and offspring attention deficit hyperactivity disorder. Sci Rep 2019; 9: 9519.

21. European Medicines Agency [cited from December $26^{\text {th }}$, 2019]. Available from: https://www.ema.europa.eu/en/ documents/prac-recommendation/prac-recommendations-signals-adopted-12-15-march-2019-prac-meeting_en.pdf.

22. Juujärvi S, Kallankari H, Pätsi P, et al. Follow-up study of the early, randomised paracetamol trial to preterm infants, found on adverse reactions at the two-years corrected age. Acta Paediatr 2019; 108: 452-58.

23. Oncel MY, Eras Z, Uras N, Canpolat FE, Erdeve O, Oguz SS. Neurodevelopmental outcomes of preterm infants treated with oral paracetamol versus ibuprofen for patent ductus arteriosus. Am J Perinatol 2017; 34: 1185-9.

24. Bhutta AT, Rovnaghi C, Simpson PM, Gossett JM, Scalzo FM, Anand KJ. Interactions of inflammatory pain and morphine in infant rats: long-term behavioral effects. Physiol Behav 2001; 73: 51-8.

25. Hooijmans CR, Rovers MM, de Vries RB, Leenaars M, Ritskes-Hoitinga M, Langendam MW. SYRCLE's risk of bias tool for animal studies. BMC Med Res Methodol 2014; 14: 43.

26. Blecharz-Klin K, Joniec-Maciejak I, Jawna K, et al. Effect of prenatal and early life paracetamol exposure on the level of neurotransmitters in rats - focus on the spinal cord. Int J Dev Neurosci 2015; 47: 13-9.

27. Blecharz-Klin K, Joniec-Maciejak I, Jawna K, et al. Developmental exposure to paracetamol causes biochemical alterations in medulla oblongata. Environ Toxicol Pharmacol 2015; 40: 369-74.

28. Blecharz-Klin K, Joniec-Maciejak I, Jawna-Zboińska K, et al. Cerebellar level of neurotransmitters in rats exposed to paracetamol during development. Pharmacol Rep 2016; 68: 1159-64.

29. Blecharz-Klin K, Piechal A, Jawna-Zboińska K, et al. Paracetamol - effect of early exposure on neurotransmission, spatial memory and motor performance in rats. Behav Brain Res 2017; 323: 162-71. 
30. Blecharz-Klin K, Wawer A, Jawna-Zboińska K, et al. Early paracetamol exposure decreases brain-derived neurotrophic factor (BDNF) in striatum and affects social behaviour and exploration in rats. Pharmacol Biochem Behav 2018; 168: 25-32.

31. Blecharz-Klin K, Wawer A, Pyrzanowska J, et al. Hypothalamus - response to early paracetamol exposure in male rat offspring. Int J Dev Neurosci 2019; 76: 1-5.

32. David A, Pancharatna K. Effects of acetaminophen (paracetamol) in the embryonic development of zebrafish, Danio rerio. J Appl Toxicol 2009; 29: 597-602.

33. Ellis LD, Berrue F, Morash M, Achenbach JC, Hill J, McDougall JJ. Comparison of cannabinoids with known analgesics using a novel high throughput zebrafish larval model of nociception. Behav Brain Res 2018; 337: 151-9.

34. Escapa C, Coimbra RN, Neupartch T, Torres T, Santos MM, Otero M. Acetaminophen removal form water by microalgae and effluent toxicity assessment by the zebrafish embryo bioassay. Water 2019; 11: 1929.

35. Hay-Schmidt A, Finkielman OTE, Jensen BAH, et al. Prenatal exposure to paracetamol/acetaminophen and precursor aniline impairs masculinisation of male brain and behavior. Reproduction 2017; 154: 145-52.

36. Klein RM, Rigobello C, Vidigal CB, et al. Gestational exposure to paracetamol in rats induces neurofunctional alterations in the progeny. Neurotoxicol Teratol 2020; 77: 106838

37. Leroux P, Hennebert C, Catteau J, et al. Neuroprotective effects vary across nonsteroidal antiinflammatory drugs in a mouse model of developing excitotoxic brain injury. Neuroscience 2010; 167: 716-23.

38. Nogueira AF, Pinto G, Correia B, Nunes B. Embryonic development, locomotor behavior, biochemical, and epigenetic effects of the pharmaceutical drugs paracetamol and ciprofloxacin in larvae and embryos of Danio rerio when exposed to environmental realistic levels of both drugs. Environ Toxicol 2019; 34: 1177-90.

39. Philippot G, Gordh T, Frediksson A, Viberg H. Adult neurobehavioral alterations in male and female mice following developmental exposure to paracetamol (acetaminophen): characterization of a critical period. J Appl Toxicol 2017; 37: 1174-81.

40. Philippot G, Hallgren S, Gordh T, Fredriksson A, Fredriksson R, Viberg H. A cannabinoid receptor type 1 (CB1R) agonist enhances the developmental neurotoxicity of acetaminophen (paracetamol). Toxicol Sci 2018; 166: 203-12.

41. Reuter I, Knaup S, Romanos M, Lesch KP, Drepper C, Lillesaar C. Developmental exposure to acetaminophen does not induce hyperactivity in zebrafish larvae. J Neural Transm (Vienna) 2016; 123: 841-8.

42. Saeedan AS, Singh I, Ansari MN, et al. Effect of early natal supplementation of paracetamol on attenuation of exotoxin/endotoxin induced pyrexia and precipitation of autistic like features in albino rats. Inflammopharmacology 2018; 26: 951-61.

43. Van den Hoogen NJ, Tibboel D, Honig WM, Hermes D, Patijn J, Joosten EA. Neonatal paracetamol treatment reduces long-term nociceptive behaviour after neonatal procedural pain in rats. Eur J Pain 2016; 20: 1309-18.

44. Viberg H, Eriksson P, Gordh T, Fredriksson A. Paracetamol (acetaminophen) administration during neonatal brain development affects cognitive function and alters its analgesic and anxiolytic response in adult male mice. Toxicol Sci 2014; 138: 139-47

45. Xia L, Zheng L, Zhou JL. Effects of ibuprofen, diclofenac and paracetamol on hatch and motor behavior in devel- oping zebrafish (Danio rerio). Chemosphere 2017; 182: 416-25.

46. Flint RB, Roofthooft DW, van Rongen A, et al. Exposure to acetaminophen and all its metabolites upon 10, 15, and $20 \mathrm{mg} / \mathrm{kg}$ intravenous acetaminophen in verypreterm infants. Pediatr Res 2017; 82: 678-84.

47. Philippot G, Nyberg F, Gordh T, Fredriksson A, Viberg H. Short-term exposure and long-term consequences of neonatal exposure to $\Delta(9)$-tetrahydrocannabinol (THC) and ibuprofen in mice. Behav Brain Res 2016; 307: 137-44.

48. Philippot G, Forsberg E, Tahan C, Viberg H, Fredriksson R. A single $\delta^{9}$-tetrahydrocannabinol (THC) dose during brain development affects markers of neurotrophy, oxidative stress, and apoptosis. Front Pharmacol 2019; 10 : 1156.

49. Scheyer AF, Melis M, Trezza V, Manzoni OJJ. Consequences of perinatal cannabis exposure. Trends Neurosci 2019; 42: 871-84.

50. Harding DR, Humphries SE, Whitelaw A, Marlow N, Montgomery HE. Cognitive outcome and cyclo-oxygenase-2 gene $(-765 \mathrm{G} / \mathrm{C})$ variation in the preterm infant. Arch Dis Child Fetal Neonatal Ed 2007; 92: F108-12.

51. Van der Veeken L, Grönlund S, Gerdtsson E, et al. Longterm neurological effects of neonatal caffeine treatment in a rabbit model of preterm birth. Pediatr Res 2020; 87: 1011-8.

52. van der Merwe J, van der Veeken L, Ferraris S, et al. Early neuropathological and neurobehavioral consequences of preterm birth in a rabbit model. Sci Rep 2019; 9: 3506.

53. Peterson RG. Consequences associated with nonnarcotic analgesics in the fetus and newborn. Fed Proc 1985; 44: 2309-13.

54. Whitelaw A, Thoresen M. Antenatal steroids and the developing brain. Arch Dis Child Fetal Neonatal Ed 2000; 83: F154-7.

55. Koo E, Oshodi T, Meschter C, Ebrahimnejad A, Dong G. Neurotoxic effects of dexmedetomidine in fetal cynomolgus monkey brains. J Toxicol Sci 2014; 39: 251-62.

56. Tamai S, Iguchi T, Niino N, et al. A monkey model of acetaminophen-induced hepatotoxicity: phenotypic similarity to human. J Toxicol Sci 2017; 42: 73-84

57. Baliga SS, Jaques-Robinson KM, Hadzimichalies NM, Golfetti R, Merrill GF. Acetaminophen reduced mitochondrial dysfunction during early cerebral postischemic reperfusion in rats. Brain Res 2010; 1319: 142-54.

58. Briyal S, Gulati A. Endothelin-A receptor antagonist BQ123 potentiates acetaminophen induced hypothermia and reduces infarction following focal cerebral ischemia in rats. Eur J Pharmacol 2010; 644: 73-9.

59. Hassell KJ, Ezzati M, Alonso-ALconada D, Hausenloy DJ, Robertson NJ. New horizons for newborn brain protection: enhancing endogenous neuroprotection. Arch Dis Child Fetal Neonatal Ed 2015; 100: F541-52.

60. Grama A, Aldea C, Burac L, et al. Acute liver failure secondary to toxic exposure in children. Arch Med Sci 2019 DOI 10.5114/aoms.2019.87716.

61. Cao H, Li H, Zhu X, et al. Three non-invasive ventilation strategies for preterm infants with respiratory distress syndrome: a propensity score analysis. Arch Med Sci 2020 DOl 10.5114/aoms.2020.93541 